\title{
The ESS neutrino super-beam near detector
}

\author{
Alexander Burgman, ${ }^{a, 1, *}$ Joochun Park, ${ }^{a, 2}$ Joakim Cederkäll ${ }^{a}$ and Peter \\ Christiansen $^{a}$ \\ ${ }^{a}$ Department of Physics, Lund University, \\ P.O Box 118, 22100 Lund, Sweden
}

E-mail: alexander.burgman@nuclear.lu.se

The ESS Neutrino Super-Beam (ESSNUSB) is a proposed long-baseline neutrino oscillation experiment, performed with a high-intensity neutrino beam, to be developed as an extension to the European Spallation Source proton linac currently under construction in Lund, Sweden. The neutrinos would be detected with the near and far detectors of the experiment, the former within several hundred meters of the neutrino production point and the latter within several hundred kilometers. The far detector will consist of a megaton-scale water-Cherenkov detector, and the near detector will consist of a kiloton-scale water-Cherenkov detector in combination with a finegrained tracking detector and an emulsion detector. The purpose of the near detector is to constrain the flux of the neutrino beam as well as to extract the electron-neutrino interaction cross-section in water, which requires high-performance energy reconstruction and particle flavor identification techniques. These measurements are crucial for the neutrino oscillation measurements that will be conducted using the far detector.

Year 2021 sees the finalization of the conceptual design of the near detector after a thorough evaluation of the performance of a number of different design options, and a characterization of the neutrino reconstruction and flavor identification performances. In this talk we report on these studies.

\footnotetext{
*** The European Physical Society Conference on High Energy Physics (EPS-HEP2021), ***

*** 26-30 July $2021 * * *$

*** Online conference, jointly organized by Universität Hamburg and the research center DESY ***
}

\footnotetext{
${ }^{1}$ For the ESSNUSB Collaboration

${ }^{2}$ Now at the Center for Exotic Nuclear Studies, Institute for Basic Science, 34126 Daejeon, Korea

* Speaker
} 


\section{Introduction}

A possible explanation for the apparent asymmetry in the matter-antimatter abundances in the Universe is a simultaneous violation of the Charge and Parity symmetries (CP) in the leptonic sector. Recent measurements by the T2K experiment, making use of the Super-Kamiokande detector, indicate a non-zero value of the leptonic $\mathrm{CP}$ violation, quantified by the $\mathrm{CP}$ violating phase $\delta_{\mathrm{CP}}$ [1]. In order to precisely measure the leptonic $\mathrm{CP}$ violation a new generation of long baseline water-Cherenkov detector -based neutrino experiments is proposed, including the European Spallation Source neutrino Super Beam (ESSNuSB) [2, 3] and the Hyper-Kamiokande experiment [4]. These experiments will also be sensitive to explore additional open questions in modern fundamental physics, such as the ordering of the neutrino mass eigenstates, proton decay, and the detection of various astrophysically produced neutrinos.

\section{The ESSNUSB experiment and near detector}

The ESS neutrino super-beam experiment is a proposed long-baseline neutrino oscillation experiment, conducted using a high-intensity beam of neutrinos produced at the European Spallation Source (ESS) in Lund, Sweden, and detected in a water-Cherenkov detector 360-540 km downstream of the source. The purpose of the ESSNUSB experiment is to measure the oscillation rates of muon-neutrinos into electron-neutrinos as well as muon-antineutrinos into electron-antineutrinos, and subsequently derive the value of the leptonic $\mathrm{CP}$-violation parameter, $\delta_{\mathrm{CP}}$.

In order to produce the high-intensity neutrino beam, several adjustments to the ESS facility are required, including an upgrade of the ESS proton linac to achieve the dedicated delivery of a $5 \mathrm{MW}$ intensity $H^{-}$ion beam with $2.5 \mathrm{GeV}$ kinetic energy and $14 \mathrm{~Hz}$ repetition rate. The ions would be stripped of all electrons and injected into a dedicated accumulator ring where the pulses are compressed to $1.1 \mu$ s in length. Four target stations receive the compressed proton bunches for collisions with a titanium bead target, thus producing a particle shower predominantly populated by charged pions. The pions are sign-selected and collimated using magnetic horns, and directed into the decay tunnel where they decay to muons and muon-neutrinos. The muons are then stopped by the beam-dump at the end of the decay tunnel, while the neutrino beam propagates towards the detectors. A small population of electron-neutrinos is also produced from muon decays into electrons. Selecting positive (negative) pions produces a beam of muon-(anti)neutrinos.

The majority of the beam neutrinos have energies between 200 and $700 \mathrm{MeV}$, with maximum intensity at $400 \mathrm{MeV}$. The beam first arrives at the near detector (ND), $250 \mathrm{~m}$ from the production point, and later at the far detector, $360-540 \mathrm{~km}$ away. The far detector is currently modeled as a $540 \mathrm{kt}$ water-Cherenkov detector situated at a depth of $1 \mathrm{~km}$ in the Swedish bedrock.

The purposes of the near detector are to measure the total neutrino flux of $\sim 10^{7}$ events per running-year ( $200 \mathrm{~d}$, equivalent to $2.16 \times 10^{23}$ protons-on-target), and to measure the interaction cross-section for electron-(anti)neutrinos incident on nuclei in water, $\sigma_{v_{e} N}$. The latter is essential for the appearance-measurement of electron-(anti)neutrinos in the far detector. The ND will consist of two main components: a fine-grained plastic-scintillator tracking detector and a cylindrical waterCherenkov detector. The tracking detector is based on the Hyper-Kamiokande Super-FGD [5], and will consist of $10^{6}$ plastic scintillator cubes arranged in a $(1.4 \times 1.4 \times 0.5) \mathrm{m}^{3}$ block. The water- 
Cherenkov detector tank will have a length of $11 \mathrm{~m}$ and a radius of $4.7 \mathrm{~m}$, and have its central axis aligned with the neutrino beam direction. The inner-surface fiducial coverage will be $30 \%$ using 3.5 inch diameter PMTs. The possibility of including a third component, based on the nuclear emulsion detector used in the NINJA experiment [6], is under investigation.

Negligible oscillation occurs before the neutrino beam arrives at the ND, yielding a beam of $>99.5 \%$ muon-neutrinos with the remainder dominated by electron-neutrinos. The low fraction of $v_{e}$ presents a challenge for measuring $\sigma_{v_{e} N}$, which requires an efficient $v_{e}$ event selection strategy.

\section{Electron-neutrino selection}

In order to develop a reliable scheme for electron-neutrino event selection a two-step process was adopted: (1) distinguishing electron events from muon events, and (2) distinguishing electronneutrino events from muon-neutrino events.

\subsection{Distinguishing electrons from muons}

In the first step, samples of electron and muon events were simulated in a model of the waterCherenkov component of the ND using the WCSIm software [7], a GEANT4 based [8-10] waterCherenkov detector simulator. The simulated charged-particle events were homogeneously and isotropically distributed in the detector tank, and uniformly distributed in kinetic energy up to $1 \mathrm{GeV}$. The events were reconstructed using the FITQUN software [11, 12], developed by the HYPERKAMIOKANDE collaboration. Several selection criteria were determined to reduce the number of misreconstructed and misidentified events, with an emphasis on the muon events that were mistakenly identified as electron events.

Sub-Cherenkov cut Rejecting muon events below the Cherenkov threshold

Reconstruction quality cut Rejecting events with poor reconstruction conditions, indicated by a low registered brightness and a reconstructed vertex position close to the detector tank wall

Cherenkov-ring resolution cut Rejecting events that are too close to the tank wall in the reconstructed propagation direction of the lepton

Vertex-reconstruction discrepancy cut Rejecting events with a large difference in vertex position and direction between the electron and muon reconstruction hypotheses

The final selection efficiency is $46.3 \%$ for electron events, with a $1.6 \%$ misidentification rate, and $43.3 \%$ for muon events with a $0.3 \%$ misidentification rate.

\subsection{Distinguishing electron-neutrinos from muon-neutrinos}

In the second step a sample of neutrino interaction vertices were simulated using the GENIE neutrino interaction generator [13-15], and were weighted to the unoscillated expected neutrino flux. The neutrino vertices were homogeneously distributed in the detector tank, using the WCSIM software, with the neutrino direction aligned with the neutrino beam axis. The numbers of expected neutrino interactions in the detector per $200 \mathrm{~d}$ running-year are shown in Table 3.1 along with the expected 
number of triggering events. The events were again reconstructed using the FITQUN software, and the selection criteria developed in the previous step were applied.

Two additional criteria were developed to address event-features that are relevant in the selection of electron-neutrino events, but inaccessible in pure charged-lepton events.

Pion-like cut Rejecting events that are identified as electrons but have a high pion likelihood

Multi-subevent cut Rejecting electron-like events with multiple identified subevents

The number of electron-identified events that remain at each selection level are shown in Table 3.2 for the four considered neutrino species. This event selection reduces the muon-neutrino event rate in the electron-like sample to a similar level as the electron-neutrino rate, for each focusing horn polarity. For the positive and negative horn polarities the signal-to-background ratios are 0.73 and 0.97 , respectively.

In order to properly measure the interaction cross-section for electron-neutrinos incident on nucleons in water, $\sigma_{v_{e} N}$, the neutrino energy must be reliably calculated. Assuming a quasi-elastic charged-current interaction, the neutrino energy $E_{v}$ is calculated using the following formula:

$$
E_{v}=\frac{m_{F}^{2}-m_{l B}^{2}-m_{l}^{2}+2 m_{B} E_{l}}{2\left(m_{B}-E_{l}+p_{l} \cos \theta_{l}\right)}
$$

Here, $m_{F}$ represents the final state mass of the nucleon, $m_{I B}=m_{I}-E_{B}$ represents the bound state energy of the target nucleon, with $m_{I}$ as the initial state free nucleon mass and $E_{B}$ as the binding energy. For the purposes of this analysis, the nucleon mass is assumed as the proton mass, and $E_{B}$ is approximated as the ${ }^{16} \mathrm{O}$ binding energy of $27 \mathrm{MeV}$. The subscript $l$ represents the final state charged lepton, and thus $m_{l}, E_{l}, p_{l}$ and $\theta_{l}$ respectively represent its mass, energy, absolute momentum and angle relative the neutrino beam axis.

\begin{tabular}{rrrrr|l}
\hline Selection level & $v_{\mu}$ & $v_{e}$ & $\bar{v}_{\mu}$ & $\bar{v}_{e}$ & Horn polarization \\
\hline Total interactions & $7.25 \times 10^{7}$ & $3.57 \times 10^{5}$ & $1.89 \times 10^{5}$ & 833 & Positive \\
Trigger & $3.81 \times 10^{7}$ & $5.61 \times 10^{4}$ & $9.09 \times 10^{4}$ & 93.5 & \\
\hline Total interactions & $6.88 \times 10^{5}$ & 4740 & $1.39 \times 10^{7}$ & $4.12 \times 10^{4}$ & Negative \\
Trigger & $3.48 \times 10^{5}$ & 645 & $6.84 \times 10^{6}$ & 5040 & \\
\hline
\end{tabular}

Table 3.1: The number of expected neutrino interactions for each neutrino flavor as well as the expected number of event triggers. Shown for both positive and negative polarization of the focusing horn.

\begin{tabular}{rrrrr|l}
\hline Selection level & $v_{\mu}\left(e^{\mathrm{ID}}\right)$ & $v_{e}\left(e^{\mathrm{ID}}\right)$ & $\bar{v}_{\mu}\left(e^{\mathrm{ID}}\right)$ & $\bar{v}_{e}\left(e^{\mathrm{ID}}\right)$ & Horn polarization \\
\hline Trigger & $1.09 \times 10^{7}$ & $5.26 \times 10^{4}$ & $2.66 \times 10^{4}$ & 88.2 & Positive \\
Charged-lepton cuts & $5.72 \times 10^{5}$ & $2.29 \times 10^{4}$ & 1430 & 35.8 & \\
Neutrino cuts & $1.50 \times 10^{4}$ & $1.10 \times 10^{4}$ & 41.1 & 32.7 & \\
\hline Trigger & $1.08 \times 10^{5}$ & 605 & $1.87 \times 10^{6}$ & 4740 & Negative \\
Charged-lepton cuts & 6720 & 259 & $5.12 \times 10^{4}$ & 2120 & \\
Neutrino cuts & 123 & 123 & 1930 & 1860 & \\
\hline
\end{tabular}

Table 3.2: The number of electron-identified events $\left(e^{\mathrm{ID}}\right)$ at each selection level for the four considered neutrino species. Shown for both positive and negative polarization of the focusing horn. 

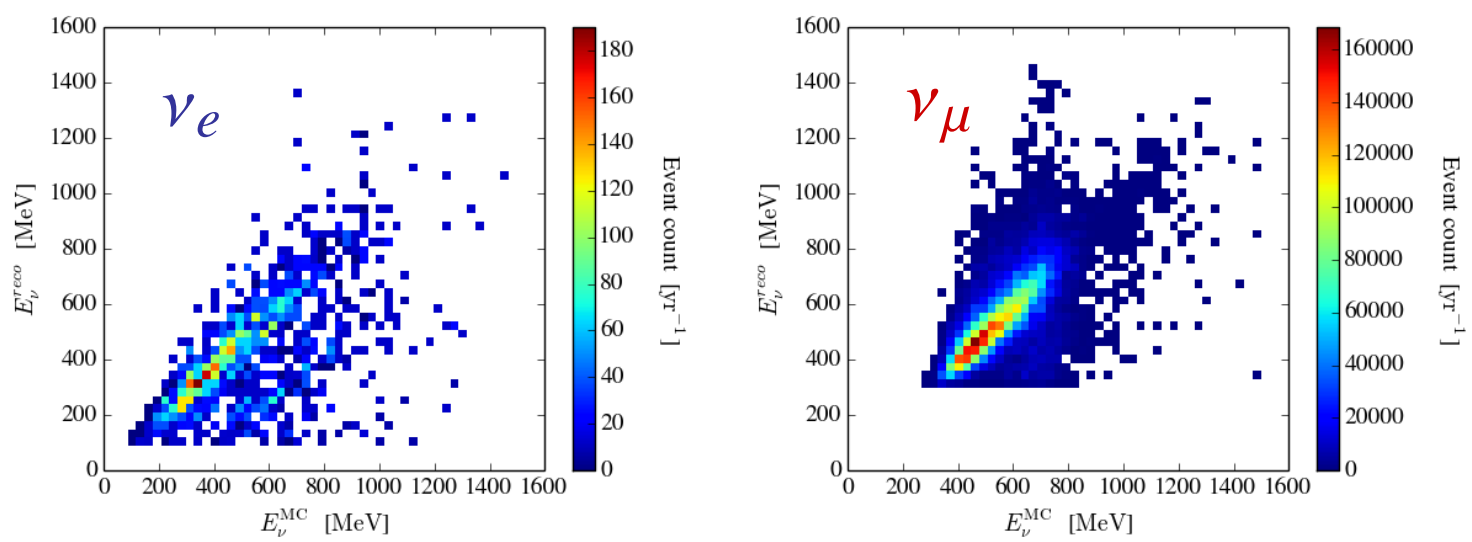

Figure 3.1: Event distributions over the true and reconstructed neutrino energies per running-year with positive horn polarity, after the full event selection. Left: electron-neutrino events. Right: muon-neutrino events.

The electron- and muon-neutrino event distributions are shown in Figure 3.1 over the true and reconstructed neutrino energies, $E_{v}^{\mathrm{MC}}$ and $E_{v}^{r e c o}$ respectively, after the full event selection. The difference between the true and reconstructed energies is distributed with root-mean-square values of $115 \mathrm{MeV}(101 \mathrm{MeV})$ for muon-neutrinos and $212 \mathrm{MeV}(138 \mathrm{MeV})$ for electron-neutrinos for positive (negative) horn polarity.

\section{Summary}

The ESSNUSB long-baseline experiment is proposed to measure the leptonic CP violation with unprecedented precision through the measurement of neutrino oscillations in a high-intensity and lowenergy neutrino beam. The neutrinos will be detected by interactions in the megaton-scale waterCherenkov far detector, as well as the near detector system. The kiloton-scale water-Cherenkov near detector will be an essential tool for measuring the electron-neutrino interaction cross-section with nucleons, for which the low $v_{e}$ fraction in the neutrino beam requires a dedicated event selection.

In this talk we have presented the current $v_{e}$ event selection strategy, where the electron-neutrino contribution was enhanced from $<2 \%$ at the trigger level to $\sim 50 \%$ at the final selection level. This selection still accepts a substantial fraction of triggered electron-neutrino events, $20 \%$ (35\%) for positive (negative) horn polarity, and demonstrates a good performance for neutrino energy reconstruction. This will enable the measurement of the neutrino-nucleon interaction cross-section.

\section{Acknowledgments}

This project received funding from the European Union's Horizon 2020 research and innovation programme under grant agreement No. 777419. Thanks are extended to C. Vilela, E. O'Sullivan, H. Tanaka, B. Quilain and M. Wilking for their assistance with using the WCSIM and FITQUN software packages. 


\section{References}

[1] T2K collaboration, Constraint on the matter-antimatter symmetry-violating phase in neutrino oscillations, Nature 580 (2020) 339 [1910 . 03887].

[2] ESSNUSB collaboration, A very intense neutrino super beam experiment for leptonic $C P$ violation discovery based on the European spallation source linac, Nucl. Phys. B $\mathbf{8 8 5}$ (2014) 127 [1309.7022].

[3] E. Wildner et al., The Opportunity Offered by the ESSNUSB Project to Exploit the Larger Leptonic CP Violation Signal at the Second Oscillation Maximum and the Requirements of This Project on the ESS Accelerator Complex, Adv. High Energy Phys. 2016 (2016) 8640493 [1510.00493].

[4] Hyper-Kamiokande collaboration, Physics potentials with the second HyPer-KamioKande detector in Korea, PTEP 2018 (2018) 063C01 [1611.06118].

[5] A. Blondel et al., A fully active fine grained detector with three readout views, JINST 13 (2018) P02006 [1707.01785].

[6] NinJa collaboration, Prospect and status of the physics run of the NINJA experiment, PoS NuFact2019 (2020) 144.

[7] "WCSIM github.” https://github.com/WCSim/WCSim.

[8] Geant4 collaboration, GEant4-a simulation toolkit, Nucl. Instrum. Meth. A 506 (2003) 250.

[9] J. Allison et al., GEANT4 developments and applications, IEEE Trans. Nucl. Sci. 53 (2006) 270.

[10] J. Allison et al., Recent developments in GEANT4, Nucl. Instrum. Meth. A 835 (2016) 186.

[11] T2K collaboration, Improving the T2K Oscillation Analysis With FITQUN: A New Maximum-Likelihood Event Reconstruction for SuPER-KAMIOKANDE, J. Phys. Conf. Ser. $\mathbf{8 8 8}$ (2017) .

[12] SuPer-Kamiokande collaboration, Atmospheric Neutrino Oscillation Analysis with Improved Event Reconstruction in SUPER-KAMIOKANDE IV, PTEP 2019 (2019) 053F01 [1901.03230].

[13] C. Andreopoulos et al., The GENIE Neutrino Monte Carlo Generator, Nucl. Instrum. Meth. A 614 (2010) 87 [0905.2517].

[14] C. Andreopoulos, C. Barry, S. Dytman, H. Gallagher, T. Golan, R. Hatcher et al., The GENIE Neutrino Monte Carlo Generator: Physics and User Manual, 1510.05494.

[15] GENIE collaboration, Neutrino-Nucleon Cross-Section Model Tuning in GENIE v3, 2104.09179. 